\title{
Formation pré- et postgraduée dans le domaine de la sécurité des patients et de la gestion des erreurs médicales
}

Groupe de travail «Sécurité des patients et gestion des erreurs médicales»
Correspondance: ASSM

Petersplatz 13

CH-4051 Bâle

Tél. 0612699030

Fax 0612699039

mail@samw.ch

\begin{abstract}
Introduction
La sécurité du patient est un problème essentiel dans nos systèmes de soins toujours plus complexes. Ce thème fait l'objet d'un regain d'attention bienvenu suite à l'intervention de partenaires influents, tels que l'Institute of Medicine aux Etats-Unis et le Comité des ministres du Conseil de l'Europe. En Suisse, un effort important d'éducation sur ce thème et celui de l'erreur médicale reste à fournir. C'est pourquoi l'Académie Suisse des Sciences Médicales a donné le mandat au présent groupe de travail «d'élaborer des propositions pour introduire la gestion des erreurs dans les formations prégraduée, postgraduée et continue de tous les professionnels de la santé, à l'intention des institutions concernées». Les conclusions principales du rapport figurent ci-après.
\end{abstract}

\section{Mandat, composition et méthodes du groupe de travail}

En 2000, l'Office fédéral de la santé publique avait interpellé l'opinion publique au moyen d'un communiqué, selon lequel peut-être environ 3000 personnes décèdent chaque année suite à des erreurs médicales, en Suisse. D'autres pays révèlent à peu près les mêmes chiffres. Bien que le système de santé soit à l'origine de nombreuses erreurs, une nouvelle conception de la gestion des erreurs médicales ne fait encore qu'émerger. Toutefois, la création de la Fondation pour la sécurité des patients représente un premier pas significatif en Suisse.

Le rapport «Buts et missions de la médecine au début du $21^{\mathrm{e}}$ siècle» publié dans le cadre du projet «L'avenir de la médecine en Suisse» proposait d'aborder la gestion des fautes professionnelles (critical incidents) et des erreurs dans le cadre des formations prégraduée, postgraduée et continue de tous les professionnels de la santé. Compte tenu de l'importance accordée à la «sécurité du patient» par la population, le groupe de pilotage du projet a décidé de traiter ce sujet dans le cadre du projet «L'avenir de la médecine en Suisse». Il a chargé un groupe de travail d'élaborer jusqu'à fin 2006, des propositions pour introduire la gestion des erreurs dans les formations prégraduée, postgraduée et continue de tous les professionnels de la santé, à l'intention des institutions concernées.

Les personnes suivantes font partie du groupe de travail:

- Prof. Arnaud Perrier, médecin-chef du Service de médecine interne générale (SMIG), HUG, Genève (responsable);

- Prof. Dieter Conen, président de la Fondation pour la sécurité des patients, Aarau;

- Marc Diby, infirmier, IPS Hôpital cantonal universitaire, Genève;

- Dr Enea Martinelli, pharmacien-chef, spitäler fmi ag, vice-président de la Fondation pour la sécurité des patients, Interlaken;

- Dr Mathieu Nendaz, membre de l'unité de développement de recherche en éducation médicale, Faculté de médecine, et médecin adjoint au SMIG, HUG, Genève;

- Lucien Portenier, bureau de l'ASI-SBK, Berne;

- Prof. Daniel Scheidegger, médecin-chef anesthésie, Hôpital universitaire, Bâle;

- Prof. Martin Täuber, doyen de la Faculté de médecine de l'Université de Berne (en tant que membre du groupe de pilotage);

- Dr Jean-Blaise Wasserfallen, direction médicale CHUV, Lausanne;

- Daniela Zahnd, bureau pour le management de la qualité, Hôpital de l'Ile, Hôpital universitaire, Berne.

Le groupe de travail a tenu quatre réunions entre le 14 février 2006 et le 4 avril 2007, date de la discussion du rapport final.

\section{Enseignement concernant la sécurité du patient: pourquoi est-ce important?}

A la suite de la publication de divers rapports alarmants aux USA et en Grande-Bretagne [1-3], la sécurité du patient est devenue une préoccupation importante. Le manque d'enseignement autour de ce thème a été relevé [4] de même que les difficultés inhérentes à la mise en place d'une telle formation [5]. En effet, le thème de l'erreur est rapidement associé à des émotions négatives 
telles que stress, colère, désespoir, ou culpabilité pouvant entraîner le déni, la déresponsabilisation ou le doute $[3,6]$, sans compter la crainte de poursuites judiciaires [7].

De plus, la formation des médecins véhicule le plus souvent le message que leur compétence future ne dépend que de la quantité de savoir acquis grâce à leur travail personnel. Ce sentiment est souvent conforté en pratique par une culture professionnelle prônant une vision du médecin comme seule source de décision par opposition à un travail d'équipe. Toute erreur sera donc ressentie comme un échec personnel et un manquement à la perfection [3]. Ce mode de penser entrave le travail d'équipe pluridisciplinaire reconnu comme condition essentielle de sécurité et de soins de qualité.

Le Comité des Ministres du Conseil de l'Europe a adopté au printemps 2006 des recommandations concernant le «Management of patient safety and prevention of adverse events in health care», dans lesquelles tout un chapitre est consacré au thème de «Patient safety education» [8]:

«Education for patient safety should be introduced at all levels within health-care systems, including individual public and private health-care organisations. The main focus should be on educating health-care professionals, including managers and senior figures involved in health-care governance, in patient-safety issues relevant to their function. In order to promote a change in attitudes towards greater patient safety, informing and educating to this end should begin for future doctors, nurses and other health professionals, and for administrators, as part of their training.»

\section{Conditions préalables à l'instauration d'un enseignement \\ concernant la sécurité des patients}

Un changement de paradigme est essentiel à l'introduction d'un enseignement sur la sécurité du patient.

Tout d'abord, à l'exemple de l'industrie de haute technologie, il faut savoir différencier l'erreur due à la personne de celle favorisée par les manquements du système dans lequel travaille l'individu. Ce changement où l'on passe de la recherche de l'individu à blâmer à celle des éléments du système qui ont favorisé l'erreur n'est pas aisé à assimiler dans nos structures. Il est néanmoins indispensable à la mise en place d'un enseignement trouvant son application en pratique.

Dans ce sens, la manière dont les cadres des institutions de soins et des organisations gèrent leurs propres erreurs et sont formés à prodiguer un feed-back constructif et explicite en cas d'erreur de leurs subordonnés joue également un rôle important $[9,10]$.
Les changements individuels de perception passent donc par une volonté institutionnelle de changement [11] et par l'introduction et le renforcement, durant la formation, du travail d'équipe, des compétences en communication, de la prise en charge de l'incertitude, et de l'évaluation des comportements professionnels [12-14].

Idéalement, cette nouvelle culture doit être introduite dès la formation prégraduée. C'est également à ce stade que des activités de formation interdisciplinaires (médecins/ soignants/pharmaciens/autres pluriprofessionnels de santé) devraient être mises en place.

Ainsi, les membres du groupe de travail sont convaincus de la nécessité:

- d'une prise de position claire des instances responsables de la formation pré- et postgraduée des différents corps de métier concernés en Suisse pour imposer ce changement;

- de la mise en place d'une politique institutionnelle de gestion des erreurs dans les diverses structures de soins, et en particulier les hôpitaux.

\section{Enseignement concernant la sécurité du patient en Suisse: état des lieux}

\section{Soins infirmiers}

Aucun module d'enseignement concernant la gestion des erreurs ne figure dans les emplois du temps des hautes écoles spécialisées, des écoles professionnelles supérieures et des centres de formation professionnelle. Des sujets isolés sont intégrés dans les formations pratiques (p.ex. la prévention des chutes, la gestion des erreurs de médication). L'institut de soins infirmiers propose des cours spécifiques par thème.

La formation en soins infirmiers est actuellement en pleine mutation. Après avoir été effectué par la Croix-Rouge suisse, le contrôle des cours de formation est maintenant assuré par l'Office fédéral de la formation professionnelle et de la technologie (OFFT). C'est pourquoi, le tableau actuel est hétérogène: il existe toujours d'un côté les écoles professionnelles de soins infirmiers selon l'ancien modèle et, d'un autre côté, notamment en Suisse romande, des hautes écoles spécialisées. En Suisse alémanique des écoles professionnelles supérieures et quelques rares hautes écoles spécialisées sont en train de voir le jour.

L'OFFT élabore actuellement un plan de formation pour les écoles professionnelles supérieures. L'ASI, représentée dans le groupe de travail, 
a préparé un projet de cours pour le domaine de la sécurité des patients.

\section{Médecine}

Selon une enquête auprès des décanats des cinq Facultés de médecine, seul Genève propose, en $4^{\mathrm{e}}$ et $5^{\mathrm{e}}$ année, un module structuré de deux heures sur les aspects éthiques et légaux des erreurs médicales. Le sujet est traité à l'aide d'un cas concret. A partir de l'automne 2007, ce thème est approfondi en $4^{\mathrm{e}}$ année (2 à 4 heures). A Berne, Bâle, Lausanne et Zurich aucun module structuré n'est proposé. Tantôt, le sujet est abordé dans le cadre de certains cours, tantôt il y a possibilité d'y réfléchir dans le cadre de l'année à choix (p.ex. à Bâle: travail avec le CIRS).

\section{Pharmacie}

Dans les études de pharmacie, ce n'est qu'en $5^{\mathrm{e}}$ année que les étudiants entrent en contact avec la pratique. Dans le module «pharmacie institutionnelle», en $5^{\mathrm{e}}$ année, la «gestion des risques» fait partie du programme. Dans la

Tableau 1

\begin{tabular}{ll}
\hline Objectifs d'apprentissage & Format \\
\hline I. Cadre général & \\
\hline Epidémiologie de l'erreur médicale & Cours/PBL* \\
\hline Définitions (erreur, événement indésirable, «near-miss», incident) & Cours/PBL \\
\hline Erreur système vs. erreur humaine & Cours/PBL \\
\hline Culture de l'erreur & Cours/PBL \\
\hline Valeurs professionnelles & Cours/PBL \\
\hline Culture du «risk management» & Cours/PBL \\
\hline Politique nationale et locale concernant la sécurité du patient & Cours/PBL \\
\hline II. Outils d'amélioration de la sécurité du patient & \\
\hline Teamwork, communication entre professionnels, briefing/debriefing & Discussion de cas/ \\
\hline Systèmes de recueil et déclaration d'incidents & jeux de rôle (méd/inf) \\
\hline Efficacité pour améliorer le système & Cours/PBL \\
\hline Outils d'analyse d'incidents («root cause analysis») & Cours/PBL \\
\hline Procédures d'amélioration & Atelier (méd/inf) \\
\hline Gestion proactive du risque et prévention & Cours/PBL \\
\hline Outils informatiques & Cours/PBL \\
\hline III. Erreurs médicales et communication & Cours/PBL \\
\hline Cadre juridique et erreur médicale & \\
\hline Obligation éthique des soignants face au patient en cas d'erreur, & \\
professionnalisme & Discussion de cas \\
\hline Communication avec le patient au sujet de l'erreur & Jeux de rôle \\
\hline Gestion pratique de l'erreur & Discussion de cas \\
\hline Politique institutionnelle et erreur & Discussion de cas \\
\hline * PBL = problem-based learning. & \\
\hline & \\
\hline
\end{tabular}

spécialisation «pharmacie d'hôpital», ce sujet est traité dans les blocs de formation «compétence sociale» et «rhétorique».

\section{Expériences d'autres groupes professionnels} D'autres groupes professionnels (compagnies aériennes, industrie chimique, banques) ont depuis longtemps de l'expérience dans le management des risques et dans la gestion des erreurs et peuvent donner des conseils judicieux concernant les instruments adéquats ou non. Il y a 15 ans déjà, le Département d'anesthésie de l'Hôpital universitaire à Bâle avait traduit et mis en application des outils pour le domaine opératoire en collaboration avec Swissair et dans des cours dirigés conjointement par un médecin et un pilote.

\section{Objectifs de formation et contenu}

\section{Objectifs généraux}

Les objectifs de formation d'un enseignement sur la sécurité du patient peuvent être résumés en trois domaines:

1. connaissances de base concernant la sécurité du patient et la gestion des erreurs;

2. compétences à travailler en équipe pluridisciplinaire;

3. compétences non techniques (communication, gestion des conflits et du stress, capacités de vigilance et d'attention) et comportement professionnel (valeurs éthiques).

\section{Contenu de l'enseignement}

Les objectifs plus détaillés qui seront décrits ici ont fait l'objet d'un consensus au sein du groupe de travail (tab. 1).

\section{Etapes de la formation}

Il convient de définir les objectifs pour les différentes étapes des formations prégraduée resp. postgraduée ainsi que pour les divers groupes professionnels. Dans le catalogue des objectifs d'enseignement de la médecine, les cours concernant la sécurité des patients font largement défaut; dans la formation en soins infirmiers, ils ne sont intégrés que partiellement.

Dans la formation prégraduée, la sensibilisation pratique à la sécurité du patient est prioritaire. L'apprentissage préventif de techniques pour la gestion des risques et des erreurs est primordial ainsi que l'internalisation d'une culture de l'erreur. Une sensibilisation à l'importance de l'interdisciplinarité doit également faire partie des objectifs prégradués. Pour les médecins, l'entrée en milieu hospitalier dans la phase clinique en $4^{\mathrm{e}} / 5^{\mathrm{e}}$ année de formation représente l'une des 
principales passerelles entre la formation théorique et la formation pratique. C'est là qu'a lieu le premier contact avec le système hospitalier.

Dans la formation postgraduée, l'établissement d'une culture de la collaboration interdisciplinaire est primordial. Par ailleurs, des discussions de cas concrets doivent permettre l'approfondissement de l'analyse des causes d'erreur et des moyens d'y remédier.

\section{Exemple de répartition possible des objectifs entre le pré- et le postgradué}

I. Prégradué

- Connaître les principes de variation et de faillibilité de tout système, y compris du système de santé.

- Connaître le mécanisme et l'impact des types d'erreurs, dont celles favorisées par le système, et les principes de leur remédiation.

- Réaliser l'importance des compétences non techniques comme l'interdisciplinarité, le travail d'équipe, l'attitude de professionnalisme, I'apprentissage de I'imprévu, la connaissance de son comportement en situation de stress et acquérir ces compétences, la communication.

- Appliquer les concepts d'analyse d'un système sur un cas (issues et procédures).

- Intégrer les liens entre un système spécifique et le système local et régional de santé.

\section{Postgradué}

- Reconnaître des situations dans I'environnement du professionnel de santé pouvant augmenter le risque d'incidents.

- Analyser une situation à risque dans son propre environnement et proposer des solutions pour le système.

- Acquérir et démontrer des compétences en gestion d'équipe, travail interdisciplinaire, et professionnalisme.

- Sensibiliser les étudiants à cette approche et servir de modèle de rôle.

- Participer activement au système de recueil et analyse d'incidents mis en place par l'institution.

\section{Formats d'enseignement}

Les formats d'enseignement doivent être adaptés aux objectifs spécifiques poursuivis (tab. 1) et au niveau de formation. L'enseignement de type cours ex cathedra doit être complété par des discussions de cas, voire des exercices pratiques, notamment pour la «root-cause analysis».
Pour prodiguer cet enseignement en pratique, plusieurs formats et outils ont été rapportés. Les patients standardisés sont utiles pour entraîner la communication avec le patient lors de l'annonce d'une erreur [15]. Les simulations de haute fidélité par mannequins [16] sont efficaces pour entraîner le travail en équipe autour d'une situation concrète, notamment de réanimation ou d'intervention chirurgicale. Les colloques d'analyse d'incidents (p.ex. morbidity-mortality conferences, CIRS, discussion de cas) [17] sont un moyen efficace de formation, à condition qu'ils se déroulent dans un climat de confiance pour ceux qui rapportent le cas. Une alternative originale est l'utilisation d'un jeu de rôle autour d'un fait réel pour éviter l'exposition directe des personnes impliquées dans une situation clinique d'erreur [5].

\section{Conditions favorables à la mise en place d'un programme d'enseignement concernant la sécurité du patient}

L'objectif ultime d'un enseignement concernant la sécurité du patient est de préparer le professionnel de santé à travailler dans un système de soins complexe, en étant conscient de son rôle et de sa responsabilité personnelle dans l'amélioration de ce système. Pour que cet objectif soit atteint, il faut, au-delà de l'enseignement luimême, qu'un certain de nombre de conditions soient réunies dans l'institution ou l'organisation de soins (culture de l'erreur). La liste ci-après détaille les conditions principales et doit permettre à chaque institution ou organisation d'évaluer si elle offre ou non des conditions propices à cet enseignement.

\section{Questions concernant les éléments qui doivent accompagner la mise en place d'un programme d'enseignement sur la sécurité du patient}

I. Formation

- Existe-t-il dans votre institution un enseignement continu et structuré dans le domaine de la sécurité du patient aux niveaux prégradué, postgradué et continu?

- Des colloques réguliers au cours desquels les incidents sont discutés sont-ils organisés (notamment des «morbidity-mortality conferences»)? Ces colloques sont-ils interdisciplinaires?

- Les cadres de l'institution ont-ils reçu une formation concernant la sécurité du patient? 
Lors de l'engagement de nouveaux cadres, les connaissances et compétences dans le domaine de la sécurité du patient sont-elles un critère de sélection? Les compétences sociales des futurs cadres sont-elles évaluées?

\section{Structures et organisation}

- Existe-t-il un système de recueil d'incidents? Les personnes qui en sont responsables sont-elles clairement identifiées?

- La politique vis-à-vis de la personne qui déclare un incident est-elle claire et explicite (approche système plutôt que sanction)?

- Y a-t-il une structure particulière pour rapporter les incidents qui peuvent avoir une portée médico-légale?

- Des bilans systématiques concernant les interventions et/ou la prise en charge des patients sont-ils organisés régulièrement (p.ex. une fois par semaine) pour vérifier s'il y a eu ou non des incidents?

- Les interventions à risque sont-elles discutées avec tous les intervenants au préalable afin d'anticiper les difficultés?

III. Leadership

- La direction de I'hôpital s'implique-t-elle dans la problématique de la sécurité du patient?

- Les chefs de service s'impliquent-ils dans la problématique de la sécurité du patient?

- Quelle est le modèle de prise de décision (autocratique ou participatif)?

\section{Instruments utiles pour développer un programme d'enseignement concernant la sécurité du patient}

Afin que le contenu de ce rapport ne reste pas qu'une déclaration d'intention, il a paru important aux membres du groupe de travail de l'accompagner d'une série d'instruments utiles. Il s'agit en particulier de matériel éducatif à disposition des enseignants (présentations PowerPoint par thème, cas à discuter, ressources bibliographiques, notamment). Ces ressources sont disponibles sur le site Internet www.patienten sicherheit.ch.

\section{Conclusion et propositions}

- La mise en place d'un enseignement concernant la sécurité du patient est indissociable d'un changement culturel sur la manière de considérer l'erreur et la profession au niveau des institutions de soins, des médecins et des éducateurs.

- Les activités pédagogiques doivent renforcer et évaluer les notions de travail en équipe pluridisciplinaire. Pour favoriser cette acquisition, il est très souhaitable que des parties de cet enseignement soient dispensées à des groupes d'étudiants interdisciplinaires (médecins, infirmiers et pharmaciens).

- Les professionnels de santé doivent être capables de communiquer au sujet de l'erreur. Les compétences non techniques indispensables (communication et comportements professionnels) doivent donc également faire partie de cet enseignement.

- L'enseignement concernant la sécurité du patient doit être intégré et mis en relation avec le curriculum existant (relation médecin-malade, gestes techniques, prescription, etc.).

- Les formats d'enseignement peuvent s'inspirer de ceux déjà décrits dans la littérature ou être créés de novo, mais ils ne peuvent à eux-mêmes remplacer la nécessité d'un indispensable changement culturel préalable.

En pratique,

- l'enseignement concernant la sécurité du patient doit désormais faire partie des objectifs de formation de toutes les filières de soignants (médecins, infirmiers, pharmaciens) tant au niveau pré- que postgradué;

- concernant les médecins, ces objectifs doivent être intégrés au catalogue suisse des objectifs qui est la référence pour les études de médecine.

\section{Références}

1 Elkin PL, Gorman PN. Continuing medical education and patient safety: an agenda for lifelong learning. J Am Med Inform Assoc. 2002;9(6 Suppl): S128-32.

2 Institute of Medicine. To err is human: building a safer health system. Washington, DC: The National Academies Press; 2000.

3 Lester H, Tritter JQ. Medical error: a discussion of the medical construction of error and suggestions for reforms of medical education to decrease error. Med Educ. 2001;35(9):855-61.

4 Pilpel D, Schor R, Benbassat J. Barriers to acceptance of medical error: the case for a teaching program. Med Educ. 1998;32(1):3-7.

5 Kirkegaard M, Fish J. Doc-U-drama: using drama to teach about patient safety. Fam Med. 2004; 36(9):628-30.

6 Engel KG, Rosenthal M, Sutcliffe KM. Residents' responses to medical error: coping, learning, and change. Acad Med. 2006;81:86-93. 
7 Wu AW, Folkman S, McPhee SJ, Lo B. Do house officers learn from their mistakes? JAMA. 1991; 265(16):2089-94

8 Comité des Ministres du Conseil de l'Europe. Recommandations «Management of patient safety and prevention of adverse events in health care». 2006.

9 Mazor KM, Fischer MA, Haley HL, Hatem D, Quirk ME. Teaching and medical errors: primary care preceptors' views. Med Educ. 2005;39(10): 982-90.

10 Mazor KM, Fischer MA, Haley HL, Hatem D, Rogers HJ, Quirk ME. Factors influencing preceptors' responses to medical errors: a factorial survey. Acad Med. 2005;80(10 Suppl):S88-92.

11 Carroll JS, Edmondson AC. Leading organisational learning in health care. Oual Saf Health Care. 2002;11(1):51-6.

12 Glavin RJ, Maran NJ. Integrating human factors into the medical curriculum. Med Educ. 2003; 37(Suppl 1):59-64.

13 Kirk LM, Blank LL. Professional behavior a learner's permit for licensure. N Engl J Med. 2005;353(25):2709-11

14 Papadakis MA, Teherani A, Banach MA, et al Disciplinary action by medical boards and prior behavior in medical school. N Engl J Med. 2005; 353(25):2673-82.
15 Battles JB, Wilkinson SL, Lee SJ. Using standardised patients in an objective structured clinical examination as a patient safety tool. Qual Saf Health Care. 2004;13(Suppl 1):i46-50.

16 Flanagan B, Nestel D, Joseph M. Making patient safety the focus: crisis resource management in the undergraduate curriculum. Med Educ. 2004;38(1): 56-66.

17 Orlander JD, Barber TW, Fincke BG. The morbidity and mortality conference: the delicate nature of learning from error. Acad Med. 2002;77(10): 1001-6.

\section{Références additionnelles}

- Barach P. Patient safety curriculum. Acad Med. 2000;75(5):551-2.

- Horsburgh M, Merry AF, Seddon M. Patient safety in an interprofessional learning environment. Med Educ. 2005;39(5):512-3.

- Madigosky WS, Headrick LA, Nelson K, Cox KR, Anderson T. Changing and sustaining medical students' knowledge, skills, and attitudes about patient safety and medical fallibility. Acad Med. 2006;81(1):94-101.

- Weingart SN, Tess A, Driver J, Aronson MD, Sands K. Creating a quality improvement elective for medical house officers. J Gen Intern Med. 2004;19(8):861-7. 